\title{
Argumentos de Densidade e o Lema de Kronecker
}

\author{
Antonio Caminha
}

\section{Resumo}

Estas notas discorrem sobre o conceito de densidade no conjunto dos reais, com especial ênfase no Lema de Kronecker e em algumas aplicações interessantes do mesmo. Em linhas gerais, seguimos a referência [1].

Palavras e frases-chave: Densidade, Lema de Kronecker, olimpíadas de matemática, problemas.

\section{Density arguments and Kronecker's lemma}

\begin{abstract}
These notes discuss the concept of density in the set of real numbers, with special emphasis on Kronecker's lemma and some of its interesting applications. In general lines we follow reference [1].

Key words and phrases: Density, Kronecker's lemma, mathematical olympiads, problems.
\end{abstract}

\section{Introdução}

Observe que o conjunto $\mathbb{Q}$ dos números racionais é espalhado por toda a reta. Quantificamos isso de maneira precisa da seguinte forma: todo intervalo $(a, b)$ da reta (com $a<b)$ contém pelo menos um (e, então, infinitos - porque?) número racional.

Uma maneira simples de ver isso é observar os conjuntos (infinitos) de números racionais $\mathbb{Z}, \frac{1}{2} \mathbb{Z}, \frac{1}{3} \mathbb{Z}, \frac{1}{4} \mathbb{Z}$, etc, onde $\frac{1}{n} \mathbb{Z}$ (para $n$ natural) representa o conjunto dos racionais que podem ser escritos com denominador $n$ (veja a figura a seguir). Realmente, como a distância entre dois números consecutivos do conjunto $\frac{1}{n} \mathbb{Z}$ é

$$
\frac{m+1}{n}-\frac{m}{n}=\frac{1}{n}
$$

$\widetilde{\mathrm{A}}$ medida que $n$ aumenta os elementos consecutivos de $\frac{1}{n} \mathbb{Z}$ vão ficando cada vez mais próximos uns dos outros, de forma que, para $n$ grande o bastante, não será possível que todos os elementos do conjunto $\frac{1}{n} \mathbb{Z}$ evitem o intervalo $(a, b)$.

Reservamos um nome específico para os conjuntos de números reais espalhados por toda a reta. 


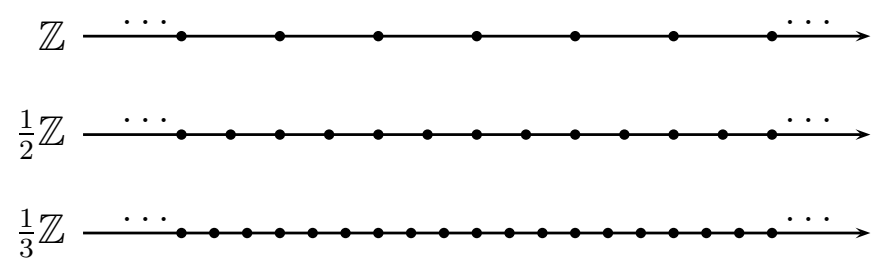

Definição 1. Um conjunto $X$ de números reais é denso em $\mathbb{R}$ se todo intervalo $(a, b)$, com $a<b$, contém pelo menos um elemento de $X$.

Evidentemente, se $X \subset \mathbb{R}$ é denso e $a<b$ são dois reais dados, então $X \cap(a, b)$ é um conjunto infinito.

Os argumentos anteriores garantem que $\mathbb{Q}$ é denso em $\mathbb{R}$. Vejamos uma aplicação simples desse fato.

Problema 2. Seja $f: \mathbb{R} \rightarrow \mathbb{R}$ uma função satisfazendo as seguintes condições:

(a) $f$ é crescente, isto é, $x<y \Rightarrow f(x)<f(y)$, para todos $x, y \in \mathbb{R}$.

(b) $f(x)=x$, para todo $x \in \mathbb{Q}$.

Mostre que $f(x)=x$, para todo $x \in \mathbb{R}$.

Solução. Suponha que exista $a \in \mathbb{R}$ tal que $f(a)<a$. Pela densidade de $\mathbb{Q}$ em $\mathbb{R}$, podemos escolher um racional $r$ tal que $f(a)<r<a$. Então, como $f$ é crescente e $f(r)=r$, temos

$$
r<a \Rightarrow f(r)<f(a) \Rightarrow r<f(a),
$$

o que contradiz a escolha $f(a)<r$.

Analogamente, também não podemos ter $f(a)>a$, de forma que a única possibilidade é que seja $f(a)=a$. Contudo, uma vez que $a \in \mathbb{R}$ foi escolhido arbitrariamente, concluímos que $f(x)=x$ para todo $x \in \mathbb{R}$.

Problema 3. Seja $f: \mathbb{R} \rightarrow \mathbb{R}$ uma função satisfazendo as seguintes condições:

(a) $f(1) \neq 0$.

(b) $f(x+y)=f(x)+f(y)$, para todos $x, y \in \mathbb{R}$.

(c) $f(x y)=f(x) f(y)$, para todos $x, y \in \mathbb{R}$.

Mostre que $f(x)=x$ para todo $x \in \mathbb{R}$. 
(Sugestão: mostre sucessivamente que $f(x)=x$ para todo $x \in \mathbb{Q}, x \neq 0 \Rightarrow f(x) \neq 0$; $x<y \Rightarrow f(x)<f(y)$. Então, aplique o resultado do problema anterior.)

O próximo problema aplica a densidade dos racionais na reta de uma forma interessante.

Problema 4 (IMO shortlist). Ache todas as funções $f: \mathbb{N} \rightarrow \mathbb{N}$ satisfazendo as seguintes condições:

(a) fé crescente.

(b) $f(1)=1$ e $f(2)=4$.

(c) $f(x y)=f(x) f(y)$ para todos $x, y \in \mathbb{N}$.

Solução. Fazendo $x=y=2$ em (c), obtemos

$$
f(4)=f(2)^{2}=4^{2} .
$$

Agora, fazendo $x=4$ e $y=2$ em (c), ficamos com

$$
f(8)=f(4) f(2)=4^{2} \cdot 4=4^{2} \cdot 2^{2}=8^{2} .
$$

Prosseguindo dessa forma, uma indução fácil nos dá $f\left(2^{k}\right)=2^{2 k}$ para todo $k \in \mathbb{N}$.

Nesse ponto, a suspeita que temos é de que deva ser $f(x)=x^{2}$ para todo $x \in \mathbb{N}$. Contudo, o que sabemos até o momento é somente que

$$
2^{k}<x<2^{k+1} \Rightarrow f\left(2^{k}\right)<f(x)<f\left(2^{k+1}\right),
$$

isto é,

$$
2^{2 k}<f(x)<2^{2(k+1)} .
$$

De todo modo, a ideia para mostrar que deve ser $f(x)=x^{2}$ é bastante simples: vamos mostrar que não pode ser $f(x) \leq x^{2}-1$ nem $f(x) \geq x^{2}+1(\operatorname{como} f(x) \in \mathbb{N}$, isso garantirá que deve ser $\left.f(x)=x^{2}\right)$.

Para o que falta, tomemos $x>1$ inteiro e examinemos o que aconteceria se tivéssemos $f(x) \leq x^{2}-1$. Nesse caso, teríamos

$$
f\left(x^{m}\right)=f(x)^{m} \leq\left(x^{2}-1\right)^{m}
$$

para todo $m \in \mathbb{N}$ (não é difícil ver que $f\left(x^{m}\right)=f(x)^{m}$ : fazendo $y=x$ em (c), temos $f\left(x^{2}\right)=f(x)^{2}$; então, fazendo $y=x^{2}$ em (c), ficamos com $f\left(x^{3}\right)=f\left(x \cdot x^{2}\right)=$ $f(x) f\left(x^{2}\right)=f(x) f(x)^{2}=f(x)^{3}$, etc $)$. 
Então, se conseguirmos garantir que existem $m, n \in \mathbb{N}$ tais que

$$
2^{n}<x^{m} \text { e }\left(x^{2}-1\right)^{m}<2^{2 n},
$$

teremos

$$
2^{2 n}=f\left(2^{n}\right)<f\left(x^{m}\right) \leq\left(x^{2}-1\right)^{m}<2^{2 n},
$$

o que será uma contradição.

As desigualdades em (1) equivalem a

$$
2^{2 n}<\left(x^{2}\right)^{m} \text { e }\left(x^{2}-1\right)^{m}<2^{2 n}
$$

ou, ainda, $\left(\operatorname{com} u=x^{2}\right)$ a

$$
(u-1)^{m}<2^{2 n}<u^{m} .
$$

A chave para encontrar $m, n \in \mathbb{N}$ tais que as últimas desigualdades acima sejam satisfeitas vem do fato de que o quociente $\frac{u^{m}}{(u-1)^{m}}$ fica muito grande $\widetilde{\mathrm{A}}$ medida que $m$ aumenta, mesmo com $\frac{u}{u-1}$ sendo somente ligeiramente maior que 1 . A fim de transformar essa intuição em um raciocínio correto, tomamos logaritmos (em qualquer base maior que 1): como $u>1$ (pois tomamos $x>1$ ), temos que

$$
\begin{aligned}
(u-1)^{m}<2^{2 n}<u^{m} & \Leftrightarrow m \log (u-1)<2 n \log 2<m \log u \\
& \Leftrightarrow \frac{\log (u-1)}{2 \log 2}<\frac{n}{m}<\frac{\log u}{2 \log 2} .
\end{aligned}
$$

Então, para garantir que $m$ e $n$ existem, basta lembrarmos que $\mathbb{Q}$ é denso em $\mathbb{R}$, de forma que o intervaio

$$
\left(\frac{\log (u-1)}{2 \log 2}, \frac{\log u}{2 \log 2}\right)
$$

contém um racional $\frac{n}{m}$.

Um argumento análogo mostra que também não podemos ter $f(x) \geq x^{2}+1$, de sorte que a única possibilidade é $f(x)=x^{2}$.

O próximo problema traz uma variação útil do argumento que estabeleceu a densidade de $\mathbb{Q}$ em $\mathbb{R}$.

Problema 5. Mostre que o conjunto dos números da forma $\frac{m}{2^{n}}$, com $m \in \mathbb{Z}, n \in \mathbb{N}$, é denso em $\mathbb{R}$.

Problema 6 (Coréia do Sul). Ache todas as funções $f: \mathbb{Z}_{+} \rightarrow \mathbb{Z}_{+}$satisfazendo as seguintes condições: 
(a) $2 f\left(m^{2}+n^{2}\right)=f(m)^{2}+f(n)^{2}$, para todos $m, n \in \mathbb{Z}_{+}$.

(b) Se $m, n \in \mathbb{Z}_{+}$, com $m \geq n$, então $f\left(m^{2}\right) \geq f\left(n^{2}\right)$.

Solução. Fazendo $n=0$ em (a), obtemos $2 f\left(m^{2}\right)=f(m)^{2}+f(0)^{2}$. Assim, segue de (b) que

$$
m \geq n \Rightarrow f(m)^{2} \geq f\left(n^{2}\right) \Rightarrow 2 f(m)^{2}-f(0)^{2} \geq 2 f(n)^{2}-f(0)^{2} \Rightarrow f(m) \geq f(n),
$$

de modo que $f$ é não decrescente.

Fazendo agora $m=n=0$ em (a), obtemos $2 f(0)=2 f(0)^{2}$, de modo que $f(0)=0$ ou 1. Consideremos esses dois casos separadamente:

i. $f(0)=1$ : fazendo $m=1$ e $n=0$ em (a), obtemos $f(1)=1$. Daí, fazendo $m=n=1$ em (a), obtemos $f(2)=1$. Suponha que já mostramos que $f\left(2^{j}\right)=1$ para todo $j<2 k$. Então, segue de (a) que

$$
2 f\left(2^{2 k}\right)=2 f\left(\left(2^{k}\right)^{2}+0^{2}\right)=f\left(2^{k}\right)^{2}+f(0)^{2}=2,
$$

de modo que $f\left(2^{2 k}\right)=1$. Se já mostramos que $f\left(2^{j}\right)=1$ para todo $j<2 k+1$, segue novamente de (a) que

$$
2 f\left(2^{2 k+1}\right)=2 f\left(\left(2^{k}\right)^{2}+\left(2^{k}\right)^{2}\right)=f\left(2^{k}\right)^{2}+f\left(2^{k}\right)^{2}=2,
$$

de modo que $f\left(2^{2 k+1}\right)=1$. Portanto, temos $f\left(2^{j}\right)=1$ para todo inteiro positivo $j$. Isto, juntamente com o fato de $f$ ser não-decrescente, garante que $f \equiv 1$.

ii. $f(0)=0$ : fazendo $m=1$ e $n=0$ em (a), obtemos $f(1)=0$ ou 2. Se $f(1)=0$, concluímos como no item anterior que $f \equiv 0$.

Suponha, pois, que $f(1)=2$, e mostremos que $f(m)=2 m$ para todo inteiro $m \geq 0$. É fácil ver, por indução por exemplo, que $f\left(2^{k}\right)=2^{k+1}$ para todo inteiro nãonegativo $k$. Por outro lado, fazendo $m=0$ na condição (a), obtemos $f(n)^{2}=2 f\left(n^{2}\right)$, de modo que $f(n)$ é par para todo inteiro positivo $n$. Como

$$
2^{n+1}=f\left(2^{n}\right) \leq f\left(2^{n}+1\right) \leq f\left(2^{n}+2\right) \leq \ldots \leq f\left(2^{n+1}-1\right) \leq f\left(2^{n+1}\right)=2^{n+2}
$$

para todo $n \geq 0$, basta mostrarmos que $f$ é crescente.

Para o que falta, tomemos inteiros não negativos $n<m$ e suponhamos que $f(m)=f(n)$. Como $f(x)^{2}=2 f\left(x^{2}\right)$ para todo $x$, é fácil provarmos por indução que, para todo inteiro não negativo $k$, tem-se $f\left(m^{2^{k}}\right)=f\left(n^{2^{k}}\right)$. A ideia, então, é escolher inteiros positivos $a, b$ e $c$ tais que $a<b$ e

$$
n^{2^{c}}<2^{a}<2^{b}<m^{2^{c}} .
$$


Uma vez feito isso, o fato de $f$ ser não decrescente dará

$$
f\left(n^{2^{c}}\right) \leq f\left(2^{a}\right)=2^{a+1}<2^{b+1}=f\left(2^{b}\right) \leq f\left(m^{2^{c}}\right),
$$

o que será uma contradição. Por fim, teremos (2) se, e só se,

$$
\log _{2} n<\frac{b}{2^{c}}<\frac{a}{2^{c}}<\log _{2} m .
$$

A possibilidade de tal escolha é possível segue do problema anterior.

Também podemos adaptar o argumento que estabeleceu a densidade de $\mathbb{Q}$ em $\mathbb{R}$ para mostrar o mesmo para o conjunto dos números irracionais.

Problema 7. Prove que o conjunto dos números irracionais é denso em $\mathbb{R}$.

Prova. Considere os conjuntos (infinitos) de números racionais $\sqrt{2} \mathbb{Z}^{*}, \frac{\sqrt{2}}{2} \mathbb{Z}^{*}, \frac{\sqrt{2}}{3} \mathbb{Z}^{*}$, $\frac{\sqrt{2}}{4} \mathbb{Z}^{*}$, etc, onde, para $n$ natural,

$$
\frac{\sqrt{2}}{n} \mathbb{Z}^{*}=\left\{\frac{m}{n} \sqrt{2} ; m, n \in \mathbb{Z}, n \neq 0\right\} .
$$

(Em palavras, o conjunto $\frac{\sqrt{2}}{n} \mathbb{Z}^{*}$ é formado pelos produtos de $\sqrt{2}$ por racionais não nulos.)

Como a distância entre dois números consecutivos do conjunto $\frac{\sqrt{2}}{n} \mathbb{Z}^{*}$ é no máximo

$$
\frac{1}{n} \sqrt{2}-\left(\frac{-1}{n} \sqrt{2}\right)=\frac{2 \sqrt{2}}{n}
$$

$\tilde{\mathrm{A}}$ medida que $n$ aumenta os elementos consecutivos de $\frac{\sqrt{2}}{n} \mathbb{Z}^{*}$ vão ficando cada vez mais próximos uns dos outros, de forma que, para $n$ grande o bastante, não será possível que todos os elementos do conjunto $\frac{1}{n} \mathbb{Z}$ evitem um intervalo dado $(a, b)$.

Essencialmente o mesmo argumento pode ser utilizado para o próximo

Problema 8. Dado um número irracional $\alpha$, mostre que o conjunto dos números reais da forma $r \alpha$, com $r \in \mathbb{Q}^{*}$, é denso em $\mathbb{R}$.

A seguir, refinamos ainda mais os argumentos acima para mostrar que certos conjuntos específicos de irracionais são densos na reta. Primeiro, um problema preliminar para entender as ideias relevantes. 
Problema 9. Mostre que existem $a, b \in \mathbb{Z}$ tais que

$$
10,4<a+b \sqrt{5}<10,401
$$

Prova. Uma saída para esse problema é baseada numa ideia esperta e em estimativas bem simples. Primeiro, admita que encontramos inteiros $c, d$ tais que

$$
0<c+d \sqrt{5}<0,001
$$

(Note que $0,001=10,401-10,4$.) Então, examinamos os múltiplos de $c+d \sqrt{5}$ :

$$
c+d \sqrt{5}, 2(c+d \sqrt{5}), 3(c+d \sqrt{5}), \ldots
$$

Dentre eles, tomemos o maior que não passa de 10,4 , digamos $k(c+d \sqrt{5})$. Então,

$$
k(c+d \sqrt{5}) \leq 10,4 \text { mas }(k+1)(c+d \sqrt{5})>10,4 .
$$

Afirmamos que $(k+1)(c+d \sqrt{5})<10,401$. Realmente, se fosse $(k+1)(c+d \sqrt{5}) \geq$ 10, 401, teríamos

$$
0,001=10,401-10,4 \leq(k+1)(c+d \sqrt{5})-k(c+d \sqrt{5})=c+d \sqrt{5}
$$

mas isso seria uma contradição a (3). Logo, basta fazer $a=(k+1) c$ e $b=(k+1) d$.

A fim de encontrar $c, d \in \mathbb{Z}$ satisfazendo (3), comecemos observando que $2<$ $\sqrt{5}<2,3$, de sorte que $0<\sqrt{5}-2<0,3$. Portanto,

$$
0<(\sqrt{5}-2)^{l}<\left(\frac{3}{10}\right)^{l}=\frac{3^{l}}{10^{l}}
$$

e esse último número é menor que $0,001=\frac{1}{1000}$ se $l \geq 6$. Isso porque

$$
\frac{3^{6}}{10^{6}}=\frac{729}{10^{6}}<\frac{10^{3}}{10^{6}}=\frac{1}{10^{3}}=\frac{1}{1000} .
$$

Assim, tomando $l=6$, temos $0<(\sqrt{5}-2)^{6}<0,001 \mathrm{e}$

$$
(\sqrt{5}-2)^{6}=\sum_{j=0}^{6}\left(\begin{array}{l}
6 \\
j
\end{array}\right) \sqrt{5}^{6-j}(-2)^{j}=c+d \sqrt{5}
$$

para certos $c, d \in \mathbb{Z}$ efetivamente calculáveis (pois $\sqrt{5}^{2 i}=5^{i} \in \mathbb{Z}$, enquanto $\sqrt{5}^{2 i+1}=$ $\left.5^{i} \sqrt{5}\right)$ 
Essencialmente a mesma ideia acima funciona para o próximo problema.

Problema 10. É dado um natural p que não é quadrado perfeito (portanto, tal que $\sqrt{p}$ é irracional). Mostre que o conjunto dos números da forma $a+b \sqrt{p}$, com $a, b \in \mathbb{Z}$, é denso em $\mathbb{R}$.

(Sugestão: comece tomando um intervalo $(\alpha, \beta)$ em $\mathbb{R}$, $\operatorname{com} \beta>0$ (o caso $\beta<0$ você analisa depois), e mostrando que basta encontrar inteiros $c$, $d$ tais que $0<c+d \sqrt{p}<$ $\beta-\alpha$. Em seguida, para encontrar tais $c, d \in \mathbb{Z}$, comece tomando $n \in \mathbb{N}$ tal que $n^{2}<p<(n+1)^{2}$; então $0<\sqrt{p}-n<1$. Como $\frac{1}{\sqrt{p}-n}>1$, podemos escrever $\frac{1}{\sqrt{p}-n}=1+q$, para algum $q>0$. Então, para $l \in \mathbb{N}$, temos

$$
0<(\sqrt{p}-n)^{l}=\left(\frac{1}{1+q}\right)^{l}=\frac{1}{(1+q)^{l}}<\frac{1}{1+l q}
$$

Mostre que podemos escolher $l$ de modo que $\frac{1}{1+l q}<\beta-\alpha$. Por fim, note que $(\sqrt{p}-n)^{l}=c+d \sqrt{p}$, para certos $c, d \in \mathbb{Z}$, e que isso resolve o problema.)

Infelizmente, com as ideias anteriores ainda não podemos mostrar que, por exemplo, o conjunto dos números da forma $a+b \sqrt[3]{5}$, com $a, b \in \mathbb{Z}$, é denso em $\mathbb{R}$. Ao tentarmos adaptar aqueles argumentos para esse caso, esbarramos no inconveniente de que o número $(\sqrt[3]{5}-1)^{l}$ não é da forma $c+d \sqrt[3]{5}, \operatorname{com} c, d \in \mathbb{Z}$.

Para contornar a situação acima, recordemos que a parte fracionária de $x$, denotada $\{x\}$, é a diferença

$$
\{x\}=x-\lfloor x\rfloor .
$$

Veja que

$$
\{x\} \in[0,1), \quad \operatorname{com}\{x\}=0 \Leftrightarrow x \in \mathbb{Z} .
$$

Problema 11. Mostre que existem $a, b \in \mathbb{Z}$ tais que

$$
10,4<a+b \sqrt[3]{5}<10,401
$$

Prova. Como no problema 9, uma vez que o intervalo de extremidades 10, 4 e 10, 401 tem comprimento 0,001, vamos nos concentrar primeiro em mostrar que existem inteiros $c$ e $d$ tais que

$$
0<c+d \sqrt[3]{5}<0,001
$$

Uma vez feito isso, o argumento terminará como fizemos no problema 9: começando $\operatorname{com} c, d \in \mathbb{Z}$ como acima, examinamos seus múltiplos:

$$
c+d \sqrt[3]{5}, 2(c+d \sqrt[3]{5}), 3(c+d \sqrt[3]{5}), \ldots
$$


Dentre eles, tomemos o maior que não passa de 10,4 , digamos $k(c+f \sqrt[3]{5})$. Então,

$$
k(c+d \sqrt[3]{5}) \leq 10,4 \operatorname{mas}(k+1)(c+d \sqrt[3]{5})>10,4
$$

$\mathrm{e}(k+1)(c+d \sqrt[3]{5})<10,401$.

Conforme já comentamos, para obter inteiros $c$ e $d$ como em (5), precisamos de uma ideia diferente da que empregamos no problema 9. Quem nos socorrerá é o princípio da casa dos pombos (PCP).

Observe que

$$
[0,1)=\left[0, \frac{1}{1000}\right) \cup\left[\frac{1}{1000}, \frac{2}{1000}\right) \cup \ldots \cup\left[\frac{999}{1000}, 1\right),
$$

e que a união do segundo membro contém 1000 intervalos. Então, dentre as 1001 partes fracionárias

$$
0,\{\sqrt[3]{5}\},\{2 \sqrt[3]{5}\},\{3 \sqrt[3]{5}\}, \ldots,\{1000 \sqrt[3]{5}\}
$$

o PCP garante que há duas, digamos $\{i \sqrt[3]{5}\}$ e $\{j \sqrt[3]{5}\}$, que pertencem a um mesmo intervalo da união anterior, digamos

$$
\{i \sqrt[3]{5}\},\{j \sqrt[3]{5}\} \in\left[\frac{k}{1000}, \frac{k+1}{1000}\right)
$$

para algum inteiro $k$ tal que $0 \leq k \leq 999$.

Assim, $|\{i \sqrt[3]{5}\}-\{j \sqrt[3]{5}\}|<\frac{1}{1000}$ e, graças a (4), podemos escrever

$$
|(i \sqrt[3]{5}-\lfloor i \sqrt[3]{5}\rfloor)-(j \sqrt[3]{5}-\lfloor j \sqrt[3]{5}\rfloor)|<\frac{1}{1000}
$$

Isso é o mesmo que

$$
|(\lfloor j \sqrt[3]{5}\rfloor-\lfloor i \sqrt[3]{5}\rfloor)+(i-j) \sqrt[3]{5}|<\frac{1}{1000}
$$

de forma que, fazendo $c^{\prime}=\lfloor j \sqrt[3]{5}\rfloor-\lfloor i \sqrt[3]{5}\rfloor$ e $d^{\prime}=i-j$, temos $c^{\prime}, d^{\prime} \in \mathbb{Z}$ e

$$
\left|c^{\prime}+d^{\prime} \sqrt[3]{5}\right|<\frac{1}{1000}
$$

Se $c^{\prime}+d^{\prime} \sqrt[3]{5}>0$, teremos obtido (5), bastando fazer $c=c^{\prime}$ e $d=d^{\prime}$. Por outro lado, se $c^{\prime}+d^{\prime} \sqrt[3]{5}<0$, então a desigualdade acima dá $-\frac{1}{1000}<c^{\prime}+d^{\prime} \sqrt[3]{5}<0$, de modo que $0<-c^{\prime}-d^{\prime} \sqrt[3]{5}<\frac{1}{1000}$; logo, obteremos (5) tomando $c=-c^{\prime}$ e $d=-d^{\prime}$. 
O tipo de argumento acima foi criado pelo matemático alemão Leopold Kronecker, na segunda metade do século XIX. O problema a seguir o generaliza.

Problema 12. Seja $\gamma$ um número irracional dado. Mostre que o conjunto dos números da forma $a+b \gamma$, com $a, b \in \mathbb{Z}$, é denso na reta.

(Sugestão: comece analisando o caso $\gamma>0$. Tome um intervalo $(\alpha, \beta)$ em $\mathbb{R}$, com $\beta>0$ (o caso $\beta<0$ você analisa depois), e mostre que basta encontrar $c, d \in \mathbb{Z}$ tais que $0<c+d \gamma<\beta-\alpha$. Para esse último passo, proceda como na discussão do problema anterior, mas dessa vez com os números $\{k \gamma\}$, com $0 \leq k \leq n$, e $n \in \mathbb{N}$ escolhido de tal forma que $\frac{1}{n}<\beta-\alpha$.)

De fato, ainda é válida a seguinte versão mais forte do problema anterior.

Teorema 13 (Lema de Kronecker). Se $\gamma$ um número irracional dado, então o conjunto dos números da forma $a-b \gamma$, com $a, b \in \mathbb{N}$, é denso na reta.

Prova. Suponha, sem perda de generalidade, que $\gamma>0$. É suficiente tomarmos $\alpha>0$ e mostrarmos que existem $a, b \in \mathbb{N}$ tais que $0<a-b \gamma<\alpha$. (De fato, uma vez feito isso, o mesmo argumento que utilizamos na primeira parte da demonstração do problema 9 garante que, dados $\alpha<\beta$ reais, existem $a, b \in \mathbb{N}$ tais que $a-b \gamma \in(\alpha, \beta)$.)

Podemos supor que $\alpha \leq 1$; basta trocar $\alpha$ por $\min \{\alpha, 1\}$ desde o início. Tome $c_{0}, d_{0} \in \mathbb{Z}$ tais que $0<c_{0}+d_{0} \gamma<\alpha$. Se $d_{0}<0$, então $c_{0}>0$ (pois $c_{0}+d_{0} \gamma, \gamma>0$ ) e nada mais há a fazer. Se $d_{0}=0$, então $0<c_{0}<\alpha \leq 1$, o que é impossível. Suponha, pois, que $d_{0}<0$. A versão mais fraca do Lema de Kronecker garante que $X=\{c+d \gamma ; c, d \in \mathbb{Z}\}$ é denso em $\mathbb{R}$. Portanto, existem inteiros $c_{n}, d_{n} \in \mathbb{Z}$, com $n \geq 1$, tais que $0<c_{n}+d_{n} \gamma<c_{0}+d_{0} \gamma$.

Se existir $n \geq 1$ tal que $d_{n}<0$ ou $d_{n}>d_{0}$, nada mais há a fazer. (No segundo caso, temos $\left(c_{0}+d_{0} \gamma\right)-\left(c_{n}+d_{n} \gamma\right) \in(0, \alpha)$ e igual a $\left(c_{0}-c_{n}\right)+\left(d_{0}-d_{n}\right) \gamma$, com $d_{0}-d_{n}<0$.) Suponha, pois, que $0 \leq d_{n} \leq d_{0}$ para todo $n \geq 1$. Então, o princípio da casa dos pombos garante a existência de índices distintos $m, n \geq 1$ tais que $d_{m}=d_{n}=d$. Supondo, sem perda de generalidade, que $c_{m}+d \gamma>c_{n}+d \gamma$, temos $0<\left(c_{m}+d \gamma\right)-\left(c_{n}+d \gamma\right)<\alpha$, isto é, $0<c_{m}-c_{n}<\alpha$. Mas, como $c_{m}-c_{n} \in \mathbb{Z}$ e $\alpha \leq 1$, isso é impossível.

Uma consequência imediata do Lema de Kronecker é que, se $\gamma$ é um número irracional dado, então o conjunto dos números da forma $-a+b \gamma$, com $a, b \in \mathbb{N}$, também é denso na reta. Realmente, se $X \subset \mathbb{R}$ é denso, então $-X:=\{-x ; x \in \mathbb{R}\}$ também é denso.

A seguir, discutimos algumas aplicações interessantes do Lema de Kronecker. 
Problema 14. Dado um intervalo $I \subset \mathbb{R}$, dizemos que $X \subset \mathbb{R}$ é denso em $I$ se $X \subset I$ e $X \cap(a, b) \neq \emptyset$, para todos $a<b$ em $I$. Prove que os conjuntos $\{\cos n ; n \in \mathbb{N}\}$ $e\{\operatorname{sen} n ; n \in \mathbb{N}\}$ são densos no intervalo $[-1,1]$.

Prova. Façamos a prova de que $\{\cos n ; n \in \mathbb{N}\}$ é denso em $[-1,1]$. A prova para o conjunto dos senos é totalmente análoga.

O conjunto $X=\{n+2 k \pi ; n \in \mathbb{N}, k \in \mathbb{Z}\}$ é denso em $\mathbb{R}$, pelo Lema de Kronecker. Sejam $\Gamma$ o ciclo trigonométrico e $A=(1,0)$. Para cada $n \in \mathbb{N}$, marque sobre $\Gamma$ o ponto $P_{n}$ tais que o arco $\widehat{A P}_{n}$, medido no sentido anti-horário a partir de $A$, tem comprimento $\ell\left(\widehat{A P_{n}}\right)=n$. Se $P \in \Gamma$ e $r>0$, a densidade de $X$ em $\mathbb{R}$ garante imediatamente que o círculo de centro $P$ e raio $r$ contém pelo menos um dos pontos $P_{n}$. Então, o conjunto formado pelas abscissas dos pontos $P_{n}$ é denso no intervalo $[-1,1]$, e tal conjunto é precisamente $\{\cos n ; n \in \mathbb{N}\}$.

Problema 15. Prove que o conjunto $\{\operatorname{tg} n ; n \in \mathbb{N}\}$ é denso em $\mathbb{R}$.

Problema 16. Mostre que existe $n \in \mathbb{N}$ tal que $\sum_{k=1}^{n} \cos ^{2} k>2020$.

(Sugestão: construa indutivamente uma sequência $n_{1}<n_{2}<\ldots<n_{m}$ de naturais tais que $\cos ^{2} n_{j}>\frac{1}{\sqrt{j}}$, para $1 \leq j \leq m$. Em seguida, mostre que $\sum_{j=1}^{m} \frac{1}{j}>2020$ para $m$ suficientemente grande, digamos $m=m_{0}$, e faça $n=n_{m_{0}}$.)

Problema 17. Se $\alpha$ é irracional, prove que os conjuntos $\{\cos (n \alpha \pi) ; n \in \mathbb{N}\} e$ $\{\operatorname{sen}(n \alpha \pi) ; n \in \mathbb{N}\}$ são densos em $[-1,1]$ e o conjunto $\{\operatorname{tg}(n \alpha \pi) ; n \in \mathbb{N}\}$ é denso em $\mathbb{R}$.

(Sugestão: estenda os argumentos dos problemas anteriores.)

Problema 18. Seja $a>1$ um inteiro que não é potência de 10, e seja s uma sequência finita de algarismos, o primeiro dos quais (ÂA esquerda) é não nulo. Prove que existe uma potência de a cuja representação decimal começa, $\tilde{A}$ esquerda, com a sequência s de algarismos.

Prova. Queremos mostrar que existem $m, n \in \mathbb{N}$ para os quais

$$
s \cdot 10^{m} \leq a^{n} \leq(s+1) \cdot 10^{m} .
$$

Tomando logaritmos em base 10, isso é o mesmo que mostrar que existem naturais $m$ e $n$ para os quais

$$
\log _{10} s+m \leq n \log _{10} a \leq \log _{10}(s+1)+m
$$


ou, ainda,

$$
\log _{10} s \leq-m+n \log _{10} a \leq \log _{10}(s+1) .
$$

Mas isso é imediato a partir do Lema de Kronecker, juntamente com o fato de que $\log _{10} a$ é irracional.

Problema 19 (A. Caminha). Prove que existem infinitos naturais $n$ tais que as representações decimais de $2^{n}$ e $5^{n}$ começam ( $\tilde{A}$ esquerda) com um 3.

Prova. A fim de que $2^{n}$ e $5^{n}$ comecem com um 3, é necessário e suficiente que existam naturais $k$ e $l$ tais que

$$
3 \cdot 10^{k}<2^{n}<4 \cdot 10^{k} \text { e } 3 \cdot 10^{l}<5^{n}<4 \cdot 10^{l}
$$

ou, tomando logaritmos em base 10, que

$$
\log _{10} 3<-k+n \log _{10} 2<\log _{10} 4 \text { e } \log _{10} 3<-l+n \log _{10} 5<\log _{10} 4
$$

Por sua vez, isso equivale a pedir que as partes fracionárias de $n \log _{10} 2$ e $n \log _{10} 5$ pertençam ao intervalo $\left(\log _{10} 3, \log _{10} 4\right)$.

Como $n \log _{10} 2+n \log _{10} 5=n$ e $\{n\}=0$, tais partes fracionárias equidistam de $\frac{1}{2}$. Então, uma vez que $\frac{1}{2} \in\left(\log _{10} 3, \log _{10} 4\right)$, com $\frac{1}{2}-\log _{10} 3<\log _{10} 4-\frac{1}{2}$, concluímos que basta mostrar que existem infinitos naturais $k$ e $n$ tais que

$$
\log _{10} 3<-k+n \log _{10} 2<1-\log _{10} 3 \text {. }
$$

Contudo, novamente pelo fato de $\log _{10} 2$ ser irracional, a existência de tais $k$ e $n$ segue do Lema de Kronecker.

Problema 20. Sejam $\alpha$ e $\beta$ irracionais tais que $\frac{\alpha}{\beta}$ também é irracional. Prove que o conjunto $\{m \alpha-n \beta ; m, n \in \mathbb{N}\}$ é denso em $\mathbb{R}$.

(Sugestão: aplique o lema de Kronecker para $\frac{\alpha}{\beta}$.)

Problema 21 (A. Caminha). Dado um natural $a>1$, mostre que existe um natural b tal que há pelo menos 2020 potências consecutivas de a entre duas potências consecutivas de $b$.

Prova. Dado $b$, queremos encontrar $a, m$ e $n$ tais que

$$
a^{m}<b^{n}<b^{n+1}<\ldots<b^{n+2019}<a^{m+1} .
$$


Para tanto, é necessário que

$$
a=\frac{a^{m+1}}{a^{m}}>\frac{b^{n+2019}}{b^{n}}=b^{2019} .
$$

Começando com um natural $a>b^{2019}$ e tomando logaritmos numa base $p>1$, queremos encontrar $m$ e $n$ naturais tais que

$$
n \log _{p} b-m \log _{p} a>0 \text { e }(m+1) \log _{p} a-(n+2019) \log _{p} b>0 .
$$

Isso é o mesmo que

$$
n \log _{p} b-m \log _{p} a \in\left(0, \log _{p} \frac{a}{b^{2019}}\right) .
$$

O resultado desejado seguirá imediatamente do problema anterior, contanto que $\log _{p} a, \log _{p} b$ e $\frac{\log _{p} a}{\log _{p} b}=\log _{b} a$ sejam irracionais. Para que $\log _{b} a$ seja irracional, basta começar com $a>b^{2019}$ primo com $b$. Para que $\log _{p} a$ e $\log _{p} b$ sejam irracionais, tome $p>a$ primo.

Terminamos estas notas com dois problemas nos quais o Lema de Kronecker funciona como coadjuvante a outras ideias.

Problema 22 (Exame Preliminar de Berkeley). Seja $f: \mathbb{R} \rightarrow \mathbb{R}$ uma função contínua, tal que $f(x)=f(x+1)=f(x+\sqrt{2})$, para todo $x \in \mathbb{R}$. Prove que $f$ é constante.

Prova. É imediato que $f(m+n \sqrt{2})=f(0)$, para todos $m, n \in \mathbb{Z}$. Dado $x \in \mathbb{R}$, tome (pelo Lema de Kronecker) sequências $\left(m_{k}\right)_{k \geq 1}$ e $\left(n_{k}\right)_{k \geq 1}$ de inteiros tais que $\lim _{k \rightarrow+\infty}\left(m_{k}+n_{k} \sqrt{2}\right)=x$. Como continuidade implica continuidade sequencial, temos

$$
f(x)=\lim _{k \rightarrow \infty} f\left(m_{k}+n_{k} \sqrt{2}\right)=\lim _{k \rightarrow \infty} f(0)=f(0) .
$$

Para o enunciado do próximo problema, denotamos por $d(X, Y)$ a distância entre os pontos $X$ e $Y$.

Problema 23 (OBM). Seja $\Pi$ o plano euclidiano e $f: \Pi \rightarrow \Pi$ uma função tal que

$$
d(P, Q)=1 \Rightarrow d(f(P), f(Q))=1,
$$

para todos $P, Q \in \Pi$. Prove que $f$ é uma isometria de $\Pi$, isto é, prove que, para todos $P, Q \in \Pi$, temos

$$
d(P, Q)=d(f(P), f(Q)) .
$$


A ideia central para a solução desse problema é mostrar que $f$ também preserva distâncias iguais a $\sqrt{3}$. Se mostrarmos isso, não será difícil mostrar que, preservando distâncias 1 e $\sqrt{3}$, a função $f$ também preservará toda distância da forma $m+n \sqrt{3}$, com $m, n \in \mathbb{Z}$ tais que $m+n \sqrt{3}>0$. Em seguida, vamos usar o Lema de Kronecker.

Prova. Para $P \in \Pi$, denotaremos $f(P)$ simplesmente por $P^{\prime}$, de forma que $d(P, Q)=$ $\overline{P Q}$ e $d(f(P), f(Q))=\overline{P^{\prime} Q^{\prime}}$. Assim, o enunciado garante que

$$
\overline{P Q}=1 \Rightarrow \overline{P^{\prime} Q^{\prime}}=1 \text {. }
$$

Afirmação $1 . \overline{P Q}=\sqrt{3} \Rightarrow \overline{P^{\prime} Q^{\prime}}=\sqrt{3}$.

De fato, dados pontos $P$ e $Q$ no plano, tais que $\overline{P Q}=\sqrt{3}$, construamos pontos $R$ e $S$ para os quais $Q R S$ e $P R S$ sejam triângulos equiláteros de lado 1 (veja a figura 1). Em seguida, giremos o losango $P R Q S$, com centro em $P$ e no sentido anti-horário,

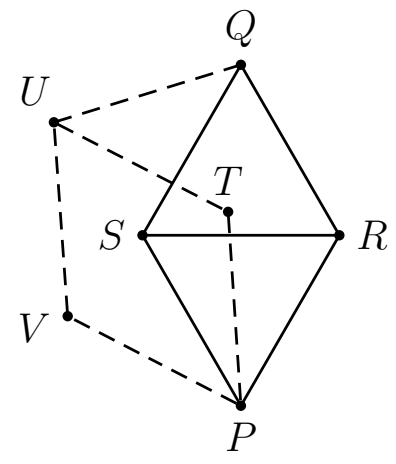

Figura 1: $\overline{P Q}=\sqrt{3} \Rightarrow \overline{P^{\prime} Q^{\prime}}=\sqrt{3}$.

até conseguirmos um losango PTUV tal que $\overline{Q U}=1$.

Observe que as imagens $P^{\prime}, R^{\prime}, S^{\prime}$ de $P, R, S$ por $f$ formam um triângulo equilátero de lado 1 (pois $f$ preserva distâncias 1). Por outro lado, como $\overline{Q^{\prime} R^{\prime}}=$ $\overline{Q^{\prime} S^{\prime}}=1$, segue que (faça uma figura para acompanhar o argumento) $Q^{\prime}=P^{\prime}$ ou $P^{\prime} R^{\prime} Q^{\prime} S^{\prime}$ é um losango congruente a $P R Q S$ (e, daí, $\left.\overline{P^{\prime} Q^{\prime}}=\sqrt{3}\right)$.

Precisamos, então, descartar a primeira possibilidade. Para tanto, basta observar que, se fosse $P^{\prime}=Q^{\prime}$, então $T^{\prime}, U^{\prime}, V^{\prime}$ seriam ao mesmo tempo pontos sobre um círculo de raio 1 centrado em $P^{\prime}=Q^{\prime}$ e vértices de um triângulo equilátero de lado 1. Isso é impossível! 
Afirmação 2. Para todo $n \in \mathbb{N}$, temos

$$
\overline{P Q}=n \Rightarrow \overline{P^{\prime} Q^{\prime}}=n .
$$

É suficiente fazer o caso $n=2$, sendo o caso geral inteiramente análogo. Sejam $P$ e $Q$ tais que $\overline{P Q}=2$, e seja $R$ o ponto médio de $P Q$, de sorte que $\overline{P R}=\overline{R Q}=1$ (veja a figura 2). Consideremos pontos $S$ e $T$ tais que $P R S, R S T$ e $Q R T$ sejam

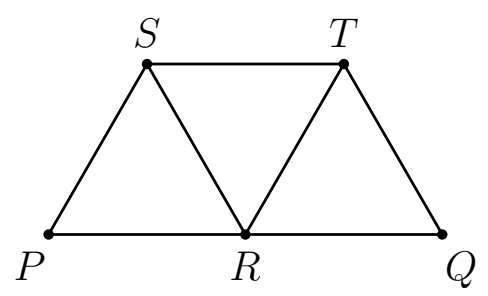

Figura 2: $\overline{P Q}=2 \Rightarrow \overline{P^{\prime} Q^{\prime}}=2$.

triângulos equiláteros de lado 1 , todos situados em um mesmo semiplano, dos determinados pela reta $\overleftrightarrow{P Q}$. Aplicando a afirmação 1 a essa configuração duas vezes (faça uma figura para acompanhar), é imediato concluir que $\overline{P^{\prime} Q^{\prime}}=2$.

De uma maneira totalmente análoga, provamos que

$$
\overline{P Q}=n \sqrt{3} \Rightarrow \overline{P^{\prime} Q^{\prime}}=n \sqrt{3} .
$$

(comece com $\overline{P Q}=2 \sqrt{3}$, marque o ponto médio $R$ de $P Q$, em seguida construa os triângulos equiláteros $P R S, R S T$ e $Q R T$, todos de lado $\sqrt{3}$, etc).

Afirmação 3. $\overline{P^{\prime} Q^{\prime}} \geq \overline{P Q}$, para todos os pontos $P, Q$ do plano.

Essa é a afirmação mais difícil da prova. Para mostrá-la, vamos usar o Lema de Kronecker, na seguinte forma refinada: seja $\overline{P Q}=l$, tal que $l$ não é nem um natural nem um real da forma $n \sqrt{3}$, para algum $n \in \mathbb{N}$. Então, podemos tomar sequências $\left(m_{k}\right)_{k \geq 1}$ e $\left(n_{k}\right)_{k \geq 1}$ de inteiros satisfazendo as seguintes condições:

i. $m_{k}<0<n_{k}$, para todo $k \geq 1$;

ii. $\lim _{k \rightarrow+\infty}\left(m_{k}+n_{k} \sqrt{3}\right)=l$;

iii. $\max \{0, l-1\}<m_{k}+n_{k} \sqrt{3}<l$, para todo $k \geq 1$. 
(Se você não se sente confortável com o conceito de limite, não se preocupe; tudo o que você precisará saber é que $\lim _{k \rightarrow+\infty} a_{k}=a$ significa que $a_{k}$ se aproxima mais e mais de $a$-tanto quanto queiramos - $\widetilde{\mathrm{A}}$ medida que $k$ aumenta mais e mais.)

A partir das condições acima, mostremos que existe um triângulo de lados $l$, $-m_{k}$ e $n_{k} \sqrt{3}$. Para tanto, como $m_{k}+n_{k} \sqrt{3}<l$, temos $l+\left(-m_{k}\right)>n_{k} \sqrt{3}$; também, $l+\left(m_{k}+n_{k} \sqrt{3}\right)>0$, o que implica $l+n_{k} \sqrt{3}>-m_{k}$; por fim, de $l-1<m_{k}+n_{k} \sqrt{3}$ segue que

$$
n_{k} \sqrt{3}+\left(-m_{k}\right)>n_{k} \sqrt{3}+m_{k}+1>l .
$$

Então, a desigualdade triangular assegura a existência de um ponto $R \in \Pi \backslash \overleftrightarrow{P Q}$ tal que $\overline{P R}=n_{k} \sqrt{3}$ e $\overline{R Q}=-m_{k}$; como já tínhamos $\overline{P Q}=l$, não há mais nada a fazer.

Segue do que fizemos acima (veja a figura 3) que $\overline{P^{\prime} Q^{\prime}}+\overline{R^{\prime} Q^{\prime}} \geq \overline{P^{\prime} R^{\prime}}$ ou, o que é o mesmo, $\overline{P^{\prime} Q^{\prime}} \geq \overline{P^{\prime} R^{\prime}}-\overline{R^{\prime} Q^{\prime}}$. Contudo, como $\overline{P^{\prime} R^{\prime}}=n_{k} \sqrt{3}$ e $\overline{R^{\prime} Q^{\prime}}=-m_{k}$, conseguimos

$$
\overline{P^{\prime} Q^{\prime}} \geq m_{k}+n_{k} \sqrt{3} .
$$

Então, como $m_{k}+n_{k} \sqrt{3} \stackrel{k}{\longrightarrow} l$, segue que

$$
\overline{P^{\prime} Q^{\prime}} \geq l=\overline{P Q} .
$$
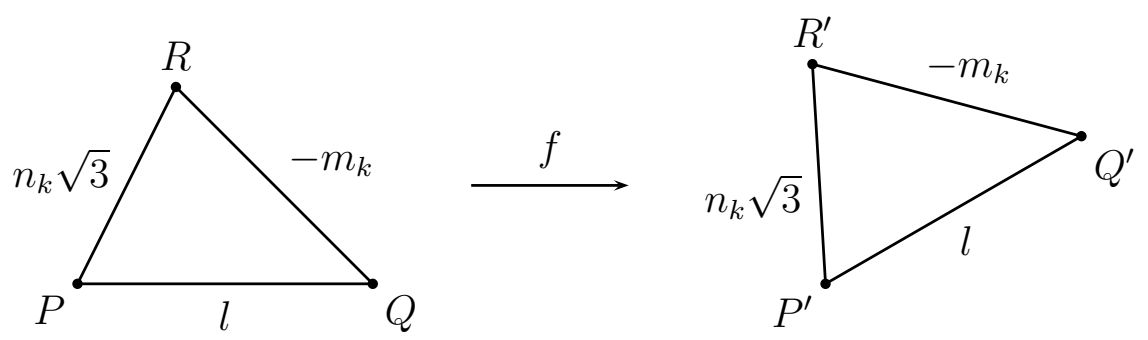

Figura 3: $\overline{P^{\prime} Q^{\prime}} \geq \overline{P Q}$.

Para terminar a demonstração do problema, consideremos novamente pontos $P$ e $Q$ no plano, com $\overline{P Q}=l$. Particione o plano em triângulos equiláteros de lado 1 , tais que um deles tenha vértice $P$ e um de seus lados situado sobre a reta $\overleftrightarrow{P Q}$ (veja a figura 4$)$.

Pelo que nós fizemos acima, as imagens, por $f$, dos vértices dessa triangulação formam os vértices de uma triangulação análoga. Por outro lado, se $X$ é um vértice 


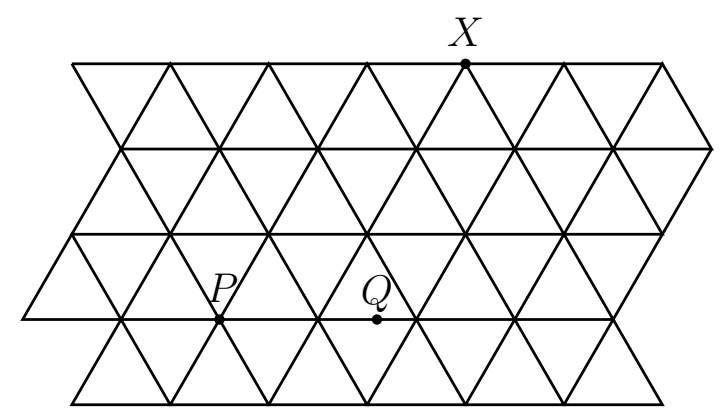

Figura $4: \overline{P Q}=l \Rightarrow \overline{P^{\prime} Q^{\prime}}=l$.

arbitrário da triangulação original (veja novamente a figura 4), então $\overline{X^{\prime} Q^{\prime}} \geq \overline{X Q}$, para todo ponto $Q$ do plano.

Geometricamente, isso significa que $Q^{\prime}$ não pertence ao interior do disco centrado em $X^{\prime}$ e tendo raio $\overline{X Q}$. Contudo, como isso é verdadeiro para todo vértice da triangulação, nós devemos necessariamente ter $\overline{P^{\prime} Q^{\prime}}=l$.

\section{Referências}

[1] A. Caminha. An Excursion Through Elementary Mathematics I. Springer Nature, Cham, 2018.

Antonio Caminha (caminha@mat.ufc.br)

Universidade Federal do Ceará, Brasil. 